\title{
High precision evaluation of the combustion enthalpy by ab-intio computations
}

\author{
Amin Alibakhshi ${ }^{1 *}$ \\ Theoretical Chemistry, Institute for Physical Chemistry, Christian-Albrechts-University, Olshausenstr. \\ 40, 24118 Kiel, Germany \\ Corresponding author: alibakhshi@pctc.uni-kiel.de
}

\begin{abstract}
Accurate evaluation of combustion enthalpy is of high scientific and industrial importance. Although via ab-initio computation of heat of reactions, as one of the promising and well-established approaches in computational chemistry, this goal should in principle be achievable, examples of reliable and precise evaluation of heat of combustion by ab-initio methods has surprisingly not yet been reported. A handful of works carried out for this purpose report significant inconsistencies between the ab-initio evaluated and experimentally determined combustion enthalpies and suggest empirical corrections to improve the accuracy of predicted data. With this background, the main aims of the present study is to investigate the reasons behind those reported inconsistencies and propose guidelines for highly accurate evaluation of combustion enthalpy via ab-initio computations. Through the provided guidelines, the most accurate results ever reported, with average absolute deviation, mean unsigned error and correlation coefficient of $1.556 \mathrm{~kJ} / \mathrm{mole}, 0.072 \%$ and 0.99999 , respectively, is achieved for theoretically computed combustion enthalpies of 40 studied hydrocarbons.
\end{abstract}

Keywords: Combustion enthalpy, Heat of reaction, ab initio, quantum mechanics

\section{Introduction}

Combustion is the key process in many important applications such as power production, transportation, heating, synthesis and processing of materials [1]. Despite being an active re- 
search area for over a century, fully understanding many aspects of the combustion processes is still a scientific challenge [2]. Unraveling these challenges sometimes requires research under special operational conditions. A well-known example is combustion experiments under microgravity conditions, which is one of the ongoing research activities in the international space station [3]. Alongside the experimental researches, theoretical studies have also substantially contributed to unraveling many of the complexities in combustion science. High-level quantum-mechanical computations have been found to be a promising tool in studying the kinetics of combustion reactions [4-6], elucidating the pathways of combustion reactions $[7,8]$ or studying combustion thermochemistry $[9,10]$.

Nevertheless, examples of successful atomic-scale computations leading to high-accuracy evaluation of combustion enthalpy as one of the most widely required features of combustion reactions are surprisingly scarce in the literature. In few studies carried out so far for this purpose, substantial inconsistencies have always been reported between the theoretically predicted and experimentally determined combustion enthalpies, as discussed in the following.

Whyman et al. [11] employed MP2 ab-initio computations to evaluate the combustion enthalpies and heats of formation for 31 compounds. Although they have not reported the accuracy of their theoretically calculated combustion enthalpies in comparison with the experimental data, their theoretically reported values demonstrate significant deviations compared to experiment, as we show in table 3 below. Audran and co-workers [12] employed ab-initio computations to calculate combustion enthalpies using four different levels of theory. They reported significant deviations between the predicted and experimental data and proposed linear relationships to empirically improve the theoretically evaluated combustion enthalpies. Mazzuca et. al. [13] studied seven ab-initio methods for evaluating the combustion enthalpies of 31 compounds. To reduce the deviations they observed between the theoretically predicted and experimentally determined combustion enthalpies, they suggested empirically scaling the theoretically predicted combustion enthalpies with scaling factors ranging from 0.9846 to 1.1866, depending on the employed level of theory. Even with this empirical scaling, they could not achieve any mean unsigned errors better than $3 \%$.

Considering that evaluation of enthalpies of chemical reactions by quantum mechanical computations is one of the most widely benchmarked and well established applications of quantum chemistry, the present study investigates the reasons behind the reported deviations between the theoretical evaluated and experimentally determined enthalpies of combustion reactions. We provide insights into appropriate treatment of the error sources and introduce guidelines for high accuracy determination of combustion enthalpies by theoretical computations. 


\section{Theoretical evaluation of combustion enthalpies}

Precise evaluation of enthalpies of gas phase reactions, as a trivial task in computational chemistry, is commonly achieved via ab-initio computation of enthalpies for individual molecules contributing to the reaction. Nevertheless, when it comes to combustion reactions, considering that the reactants and products might not always be in the gaseous state, phase change thermodynamics can also play an important role and should be taken into account, which significantly adds to the computational challenges. In our literature survey, however, we noticed that the phase change enthalpies are commonly overlooked, which is one of the main contributors to the reported inconsistency between the theoretically evaluated and experimentally determined combustion enthalpies, as demonstrated in the results section.

For appropriate treatment of phase change thermodynamics in combustion reactions, careful attention should be paid to differences in defining the state of reactants and products and to different conventions in reporting combustion enthalpies. The experimentally determined combustion enthalpy data, commonly measured by oxygen bomb calorimetry, are typically reported either as gross or net heat of combustion. Gross heat of combustion refers to the total amount of heat released in the calorimetry experiment, where both the reactants and products are nearly at room temperature and in their standard states [14]. On contrast, for the net heat of combustion, while the reactants are considered in their standard states, the combustion products are assumed to be in the gas phase [14]. Clearly, the most obvious deviation between the reported gross and net heats of combustion is due to the heat released by condensation of water molecules produced in the combustion reaction. It occurs as a result of cooling down the combustion products by the heat bath in which the combustion chamber is placed during the calorimetry measurement.

In addition to phase change thermodynamics, inaccuracies in theoretical computations as well as experimentation can both significantly influence the accuracy of the obtained results. One of the most influencing parameters is the accuracy of employed level of theory. Additionally, inappropriate energy minimization as the mandatory step before carrying out ab-initio calculation of thermal energy, can remarkably reduce the accuracy of obtained results. The main aim of the present study is to investigate and benchmark the impact of such intricacies and demonstrate appropriate employment of ab-initio computations for high accuracy evaluation of combustion enthalpy. 


\section{Computational details}

The experimentally determined gross heats of combustion for 40 hydrocarbons reported by Walters [15] were used as reference combustion enthalpies. The full list of the studied hydrocarbons is reported in table 1 . The selected hydrocarbons only contain $\mathrm{C}, \mathrm{H}$ and $\mathrm{O}$ atoms, to avoid complications e.g. due to solvation of nitric or sulfuric acid in water, produced by combustion of molecules containing nitrogen or sulfur and leading to contributions to the measured combustion enthalpy [14].

Evaluation of in vacuo enthalpies of compounds was carried out by normal mode analysis based on the rigid rotor harmonic oscillator approximation, as a standard approach for evaluation of thermodynamic quantities in theoretical chemistry [16]. To that end, for each compound we first optimized the three-dimensional geometries of the molecules in vacuo. These optimized structures were then used to calculate the ground state electronic energies and normal mode vibrational frequencies required for calculating thermal effects.

Considering that the molecular configurations found at this stage by geometry optimization can yield wide range of energies, appropriate geometry optimization plays a significant role in the ab-inito-evaluated molecular enthalpies and hence the resulting combustion enthalpy, as discussed in the results reaction and demonstrated in figure 2. Accordingly, trying to search for geometries yielding the global minimum on the potential energy surface should be attempted during the geometry optimization. To that end, we considered multi-start geometry optimization using 20 different initial structures generated via the genetic algorithm module of the open babel toolbox [17]. The configuration which yielded the lowest energy after optimization was then used for calculation of the combustion enthalpy.

Quantum-mechanical (QM) computations were carried out at the DSD-PBEP86-D3/ Def2QZVP level of theory, which is one of the most accurate methods for thermochemistry evaluations [18]. To study the influence of the employed level of theory on the accuracy of the obtained results, we also evaluated the molecular enthalpies at the B3LYP/6-311+G(2d,p) level of theory.

Considering that the theoretically evaluated enthalpies are computed for molecules in vacuo, for non-gaseous reactants, the QM evaluated enthalpy of the reactants in their standard state was obtained by subtracting their heat of phase change from the standard state to the gas phase from the initially computed in vacuo enthalpies. Although for that purpose, the thermodynamic quantities in the condensed phase can be theoretically evaluated either via the implicit solvent approaches [19], statistical thermodynamics models [20] or empirically applied through machine learning [21], a more straightforward way to take them into account is via the experimental data of phase change. To that end, the phase change enthalpies were taken from the NIST database. Similarly, considering that the reference data used in the 
present study are gross heats of combustion, the vaporization enthalpy of water with the value of $43.898 \mathrm{~kJ} / \mathrm{mol}[22]$ was also subtracted from the QM evaluated enthalpy of water in vacuo to yield the QM enthalpy of water in the liquid state. In calculation of the enthalpy of $\mathrm{O} 2$ molecules, we considered the triplet state as the ground electronic state, as conventionally employed in theoretical evaluations of combustion enthalpy[11]. At the DSD-PBEP86-D3/ Def2QZVP level of theory, our QM computation of the ground state energies with zero point energy included resulted in -150.129205 and -150.179060 Hartree per molecule for the singlet and triplet multiplicity states of $\mathrm{O} 2$, respectively, which implies that the triplet state should indeed be considered as the ground state. Since measurement of combustion enthalpies is commonly carried out under 30 bar pressure [14], we also evaluated the pressure impacts on the enthalpy of the studied compounds. To that end, we exploited the following fundamental thermodynamics relationship:

$$
\left(\frac{\partial H}{\partial P}\right)_{T}=V+T\left(\frac{\partial S}{\partial P}\right)_{T}=V-T\left(\frac{\partial V}{\partial T}\right)_{P} .
$$

Considering that the changes in thermal expansion of solids and liquids for increasing the pressure from 1 to 30 bar is negligible, we only evaluated the pressure impacts on the enthalpies of gaseous compounds. To that end, the molar volume of gaseous compounds and their derivative with respect to temperature required by Eq. (1) were evaluated via the Redlich-Kwong equation of state defined as [23]:

$$
\begin{aligned}
P & =\frac{R T}{V-b}-\frac{a}{\sqrt{T} V(V+b)}, \\
a & =0.42748 \frac{R^{2} T_{c}^{2.5}}{P_{c}}, \\
b & =0.08664 \frac{R T_{c}}{P_{c}},
\end{aligned}
$$

where $R$ is the universal gas constant, $V$ is the molar volume, and $T_{c}$ and $P_{c}$ are the critical temperature and pressure, respectively. Accordingly, for pressures from 1 to 30 bar with 1 bar intervals, the molar volumes were calculated for temperatures from $280 \mathrm{~K}$ to $320 \mathrm{~K}$ with $1 \mathrm{~K}$ intervals via solving eq. (2) by the bisection method. Using the calculated $V-T$ values for each pressure, a third-order polynomial was fitted and used to calculate the partial derivative $\frac{\partial V}{\partial T}$ as required by eq. (1). Using the calculated molar volumes and $\frac{\partial V}{\partial T}$ for all pressures, the pressure-induced enthalpy changes were calculated by numerically evaluating the following integral: 


$$
\Delta H=\int_{1}^{30}\left(V-T\left(\frac{\partial V}{\partial T}\right)_{P}\right) d p .
$$

The accuracies of the predicted combustion enthalpies are reported as Average Absolute Deviation $(A A D)$ and percentage Average Absolute Relative Error ( $M U E \%)$, defined as:

$$
\begin{aligned}
& A A D=\frac{1}{N} \sum\left(\left|y_{i}^{\text {exp }}-y_{i}^{\text {pred }}\right|\right), \\
& A A R E \%=\frac{1}{N} \sum\left(\left|\frac{y_{i}^{\text {exp }}-y_{i}^{\text {pred }}}{y_{i}^{\text {exp }}}\right|\right) \times 100
\end{aligned}
$$

All the computations were carried out in Gaussian 16 software [24] on the High Performance Computing center clusters of the Christian-Albrechts-University of Kiel.

\section{Results and discussions}

The details of the computed molecular enthalpies at the DSD-PBEP86-D3/ Def2QZVP level of theory based on the previously discussed recipes are reported in table 1, together with a direct comparison to experiment. Also, for the same level of theory the calculated enthalpies for $\mathrm{H}_{2} \mathrm{O}, \mathrm{CO}_{2}$ and $\mathrm{O}_{2}$, as molecules involved in all combustion reactions, are reported in table 2 .

Using the QM-evaluated enthalpies corrected for phase change enthalpies of water and reactants, the predicted combustion enthalpies yielded AAD, AARE\% and correlation coefficient of $17.68 \mathrm{~kJ} / \mathrm{mole}, 0.623 \%$ and 0.99999 , respectively. These results, which are directly calculated by ab-initio computation without any further correction, show a remarkable improvement compared to results reported elsewhere. For example, the theoretically calculated combustion enthalpies reported by Mazzuca et. al. [13] yielded a AARE of roughly 3\%, even after being empirically scaled. According to these results, taking into account the pressure and non-ideality impacts the predictability of combustion enthalpy only slightly.

To further improve the accuracy of the theoretically computed combustion enthalpies, using the experimentally determined heats of combustion and QM predicted enthalpies of the reactants, we computed the optimum values of enthalpies for $\mathrm{H}_{2} \mathrm{O}, \mathrm{CO}_{2}$ and $\mathrm{O}_{2}$ molecules which yielded the minimum AAD. Comparing these values with the theoretically computed enthalpies reported in table 2 shows excellent agreements, with negligible percentage deviations. 
Table 1 Details of the theoretically calculated and experimental data.

\begin{tabular}{|c|c|c|c|c|c|c|c|}
\hline Compound & Std. & $\Delta \mathbf{H}_{\text {std-gas }}$ & $\mathrm{H}_{\mathrm{QM}, \text { reactant }}$ & $\Delta \mathrm{H}_{\text {comb.,QM }}$ & $\Delta \mathrm{H}_{\text {comb.,QM,opt,P }}$ & $\Delta \mathrm{H}_{\text {comb.,QM,opt }}$ & $\Delta \mathbf{H}_{\exp }$ \\
\hline Oxyrane & $\mathrm{g}$ & 0 & -403132.59 & -1312.7 & -1305.6 & -1305.54 & -1305.53 \\
\hline Cyclopentane & 1 & 28.8 & -514947.83 & -3307.8 & -3288.88 & -3288.78 & -3288.85 \\
\hline Ethylbenzene & 1 & 41 & -814646.57 & -4599.2 & -4561.19 & -4561.57 & -4561.44 \\
\hline 2-Butanone & 1 & 34 & -609289.96 & -2457.59 & -2442.73 & -2442.82 & -2442.95 \\
\hline Methanol & 1 & 37.6 & -303387.53 & -727.72 & -726.78 & -726.76 & -726.47 \\
\hline Cyclobutane & $\mathrm{g}$ & 0 & -411847.57 & -2756.94 & -2742.04 & -2741.72 & -2742.09 \\
\hline Acetone & 1 & 31.27 & -506296.27 & -1800.15 & -1789.07 & -1789.19 & -1789.6 \\
\hline Dimethyl ether & $\mathrm{g}$ & 0 & -406302.62 & -1463.75 & -1459.25 & -1458.99 & -1459.71 \\
\hline 2-Propanol & 1 & 45 & -509403.24 & -2014.26 & -2005.76 & -2005.69 & -2004.92 \\
\hline Ethane & g & 0 & -209058.72 & -1564.61 & -1557.61 & -1559.4 & -1558.59 \\
\hline Acetaldehyde & $\mathrm{g}$ & 0 & -403245.3 & -1199.99 & -1192.91 & -1192.83 & -1191.93 \\
\hline Cyclopropane & $\mathrm{g}$ & 0 & -308851.28 & -2102.1 & -2090.96 & -2090.69 & -2089.79 \\
\hline Formic acid & 1 & 46.3 & -497680.78 & -256.43 & -253.19 & -253.52 & -254.46 \\
\hline Ethanol & 1 & 42.3 & -406394.38 & -1371.99 & -1367.28 & -1367.23 & -1366.23 \\
\hline Butane & $\mathrm{g}$ & 0 & -415038.88 & -2886.7 & -2874.42 & -2873.89 & -2874.96 \\
\hline Ethyl acetate & 1 & 35 & -806639.85 & -2250.74 & -2236.14 & -2236.42 & -2237.68 \\
\hline Isopropyl benzene & 1 & 44 & -917644.63 & -5252.27 & -5210.48 & -5210.83 & -5212.17 \\
\hline Diethyl ether & 1 & 27.1 & -612335.26 & -2733.37 & -2721.09 & -2721 & -2722.42 \\
\hline Benzene & 1 & 33.9 & -608647.31 & -3296.2 & -3265.76 & -3266.18 & -3264.75 \\
\hline 1,4-Dioxane & 1 & 38 & -806515.02 & -2375.57 & -2360.98 & -2361.25 & -2362.73 \\
\hline 1,2-Ethanediol & 1 & 65 & -603714.14 & -1195.28 & -1190.83 & -1190.97 & -1189.44 \\
\hline Phenol & $\mathrm{s}$ & 69.7 & -806003.58 & -3082.98 & -3052.81 & -3053.41 & -3051.84 \\
\hline vinyl acetate & 1 & 37.2 & -803470.61 & -2098.9 & -2081.72 & -2082.18 & -2080.62 \\
\hline Propanol & 1 & 47 & -509388.49 & -2029.01 & -2020.52 & -2020.45 & -2018.73 \\
\hline Heptane & 1 & 36 & -724039.74 & -4839.22 & -4815.32 & -4814.99 & -4813.15 \\
\hline Cyclohexane & 1 & 33.1 & -617968.58 & -3938.18 & -3915.48 & -3915.35 & -3917.19 \\
\hline 1-pentanol & 1 & 57 & -715372.87 & -3346.88 & -3330.82 & -3330.71 & -3328.86 \\
\hline Glycerol & 1 & 91.7 & -904045.28 & -1658.31 & -1650.35 & -1650.64 & -1652.52 \\
\hline Propane & $\mathrm{g}$ & 0 & -312048.73 & -2225.73 & -2217.21 & -2216.71 & -2218.62 \\
\hline Acetic acid & 1 & 50.3 & -600705.65 & -882.69 & -875.67 & -875.98 & -874.05 \\
\hline Pentane & 1 & 26.5 & -518055.34 & -3521.37 & -3505.03 & -3504.74 & -3506.75 \\
\hline Isopropyl ether & 1 & 32.26 & -818344.07 & -4026.81 & -4006.96 & -4006.83 & -4008.9 \\
\hline Furan & 1 & 27.71 & -603001.12 & -2104.27 & -2084.24 & -2084.7 & -2082.44 \\
\hline Toluene & 1 & 37 & -711652.04 & -3942.6 & -3908.38 & -3908.77 & -3906.28 \\
\hline Hexane & 1 & 31 & -621049.89 & -4177.95 & -4157.83 & -4157.52 & -4160.07 \\
\hline 1-Methylnaphthalene & 1 & 59 & -1114374 & -5861.91 & -5804.81 & -5805.67 & -5808.23 \\
\hline Benzaldehyde & 1 & 48 & -905857.39 & -3559.21 & -3522.68 & -3523.44 & -3526.08 \\
\hline Cyclohexene & 1 & 33.57 & -614808.73 & -3776.95 & -3751.67 & -3751.73 & -3748.59 \\
\hline 1-Butene & $\mathrm{g}$ & 0 & -411869.34 & -2735.16 & -2720.3 & -2719.94 & -2715.74 \\
\hline m-Cresol & 1 & 60 & -908995.93 & -3741.76 & -3707.8 & -3708.38 & -3702.26 \\
\hline
\end{tabular}

All enthalpies are in $\mathrm{kcal} / \mathrm{mole}$

The columns from left to right represent:

Std.: standard state ( gas $=\mathrm{g}$, liquid $=1$, solid $=\mathrm{s}$ )

$\Delta \mathrm{H}_{\text {std-gas: }}$ The enthalpy of phase change from the standard state to the gas phase

$\mathrm{H}_{\mathrm{QM}}$,reactant: The QM enthalpies of individual reactants in the gas phase

$\Delta \mathrm{H}_{\text {comb.,QM: }}$ Combustion enthalpy directly obtained via $\mathrm{QM}$ enthalpy of reaction

$\Delta \mathrm{H}_{\text {comb.,QM,opt,P: }}$ Combustion enthalpy corrected for pressure impacts using optimized enthalpies for $\mathrm{H} 2 \mathrm{O}, \mathrm{CO} 2$ and $\mathrm{O} 2$ and $\mathrm{QM}$ enthalpy of reactants

$\Delta \mathrm{H}_{\text {comb.,QM,opt: }}$ Combustion enthalpy without pressure correction using optimized enthalpies for $\mathrm{H} 2 \mathrm{O}, \mathrm{CO} 2$ and $\mathrm{O} 2$ and $\mathrm{QM}$ enthalpy of reactants

$\Delta \mathrm{H}_{\exp }$ : The experimentally determined data 
Table 2 QM calculated and optimum enthalpies of $\mathrm{H}_{2} \mathrm{O}, \mathrm{CO}_{2}$ and $\mathrm{O}_{2}$.

\begin{tabular}{llll}
\hline & $\mathrm{H}_{2} \mathrm{O}(\mathrm{l})$ & $\mathrm{CO}_{2}(\mathrm{~g})$ & $\mathrm{O}_{2}(\mathrm{~g})$ \\
\hline QM & -200464.123 & -494616.132 & -394286.085 \\
Optimized & -200466.979 & -494610.356 & -394286.663 \\
& & & \\
\hline
\end{tabular}

Using the optimized enthalpies of $\mathrm{H}_{2} \mathrm{O}, \mathrm{CO}_{2}$ and $\mathrm{O}_{2}$ and $\mathrm{QM}$ evaluated values for the reactants, we could theoretically reproduce the experimentally determined combustion enthalpies with AAD, AARE\% and correlation coefficient of $1.556 \mathrm{~kJ} / \mathrm{mole}, 0.072 \%$ and 0.99999, respectively, which are the most accurate results for evaluation of combustion enthalpy ever reported, to the best of our knowledge. A graphical comparison of the theoretically evaluated and experimentally determined combustion enthalpies is depicted in figure 1 .

As can be inferred from the above-mentioned results, even the very slight deviations in the QM calculated enthalpies of $\mathrm{H}_{2} \mathrm{O}, \mathrm{CO}_{2}$ and $\mathrm{O}_{2}$ from the optimum values lead to an increase of $16.124 \mathrm{~kJ} / \mathrm{mol}$ in the obtained AAD. This implies that the accuracy of the employed computational method plays the key role in high-precision evaluation of the combustion enthalpies and should be carefully considered.

This can also be inferred from table 3 , which provides a comparison of theoretically predicted combustion enthalpies for some combustion reactions in the gas phase reported in the literature with our results. These results clearly show large deviations between the results obtained via different levels of theory. While the results at the MP2 level of theory typically yield more satisfactory results, other levels of theory clearly yield inappropriate estimations of molecular enthalpies.

To further demonstrate the importance of applied level of theory, we also computed the combustion enthalpies at the B3LYP/6-311+G(2d,p) level of theory for the same dataset and computational details.

According to these results, for computations at B3LYP /6-311+G(2d,p) level of theory and after re-optimizing the enthalpies of $\mathrm{H}_{2} \mathrm{O}, \mathrm{CO}_{2}$ and $\mathrm{O}_{2}$, we obtained $\mathrm{AAD}$ and AARE\% of $16.239 \mathrm{~kJ} / \mathrm{mol}$ and $0.825 \%$, respectively. Without optimizing the enthalpies of $\mathrm{H}_{2} \mathrm{O}, \mathrm{CO}_{2}$ and $\mathrm{O}_{2}$, the original QM computations yielded AAD and AARE\% of $130.030 \mathrm{~kJ} / \mathrm{mol}$ and $5.24 \%$. Therefore, the results obtained via the B3LYP $/ 6-311+\mathrm{G}(2 \mathrm{~d}, \mathrm{p})$ level of theory are roughly one order of magnitude less accurate than those obtained via DSD-PBEP86-D3/ Def2QZVP level of theory.

By analyzing the details of computed energies we noticed that molecular thermal energies, i.e. the kinetic energy due to rotation and translation energy and vibrational energies, 


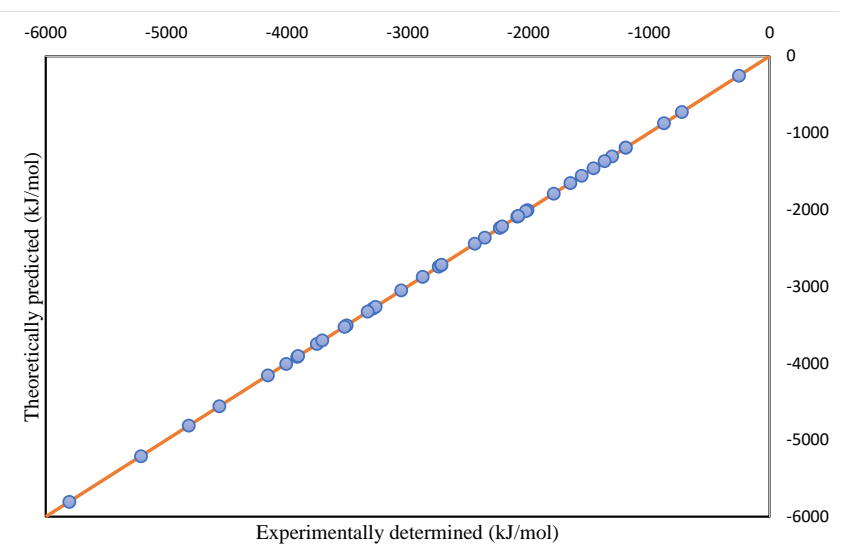

Fig. 1 comparison of theoretically predicted and experimentally determined combustion enthalpies.

contribute on average only $0.625 \%$ and $0.541 \%$ to the computed combustion enthalpies, while the changes in ground state electronic energies of reactants and products are the main contributions to the heat released by combustion. Accordingly, the accuracy of the employed level of theory in reproducing the ground state electronic energy and not the thermal effects play the key role in the accuracy of the obtained results. By comparing the thermal and electronic energies evaluated via DSD-PBEP86-D3/ Def2QZVP and B3LYP /6-311+G(2d,p) levels of theory, we observed an AAD of $148.425 \mathrm{KJ} / \mathrm{mol}$ between the computed ground state electronic energies, while for thermal energies the AAD was only $0.781 \mathrm{KJ} / \mathrm{mol}$. This is because the theoretical methods are usually parameterized using the quantities which are related to the thermal energies, such as vibrational frequencies or thermodynamic quantities, while the absolute values of ground state energies are not experimentally measurable. These results also reveal why the accuracy of theoretical methods for combustion reactions is so different from the benchmark results obtained for other case studies. The reason is that the large amount of energy released by combustion reactions is mainly due to electronic energies, which implies substantial deviations between electronic energies of reactants and products.

The DSD-PBEP86-D3/Def2QZVP level of theory used in the present study supersedes most of the conventionally accepted functionals in studying thermochemistry and for reproducing the thermal effects [25]. However, in terms of accuracy for reproducing ground state electronic energy this method still has slight inaccuracies compared to the high level and computationally demanding methods such as $\operatorname{CCSD}(\mathrm{T})$, which is commonly considered as the gold standard in theoretical chemistry [26].

Based on all these observations, for the most reliable evaluation of combustion enthalpies in which a substantial difference between the ground state energies of reactants and products exists, employing methods which are specifically parameterized for reproducing ground state 
electronic energies like CCSD or DLPNO-CCSD(T), which is a cost-effective method for accurate reproduction of $\mathrm{CCSD}(\mathrm{T})$ energies [27] for estimating ground state energies, and using other common methods for evaluating the thermal effects is recommended.

After the accuracy of the employed level of theory, the second most important source of inaccuracy in theoretically evaluated combustion enthalpies arises from inappropriately optimized structures with high energies. As for almost all poly-atomic molecules, several local minima exist on the potential energy surface, geometry optimizations started from different initial structures can result in quite diverse geometries and molecular energies and consequently different predicted combustion enthalpies. As an example, theoretical computations on the two structures of acetic acid depicted in figure 2, which were obtained by geometry optimizations started from different initial structures, yield quite different combustion enthalpies. While QM computations for the low-energy structure (without optimizing the enthalpies of $\mathrm{H}_{2} \mathrm{O}, \mathrm{CO}_{2}$ and $\mathrm{O}_{2}$ yields a combustion enthalpy with 8.64 $\mathrm{kJ} /$ mole absolute error, the same computation for the high-energy structure results in 29.76 $\mathrm{kJ} /$ mole absolute error.

Inaccuracies from inappropriately optimized structures can be avoided by employing efficient general global optimization algorithms [28-30] or rotamer searches [31] or, for small molecules as those considered here, using multi-start optimization. In the present study we selected the latter approach as discussed in the previous section.
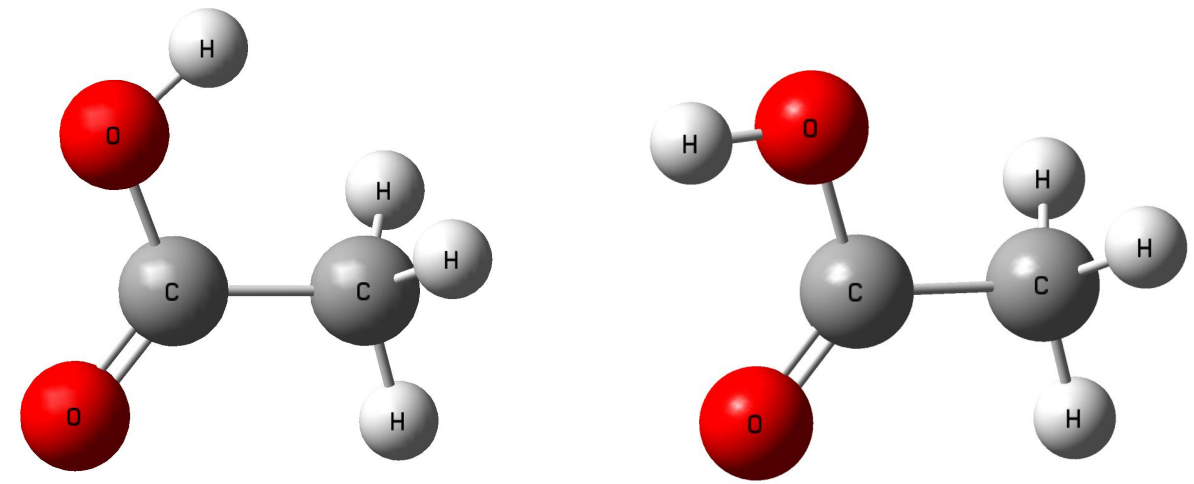

Fig. 2 Two locally optimized structures of the acetic acid yield gas phase QM calculated enthalpies of $-600634.230 \mathrm{~kJ} / \mathrm{mole}$ (left) and $-600655.349 \mathrm{~kJ} / \mathrm{mole}$ (right).

Yet another reason of deviation between the QM predicted and optimum enthalpies can be overlooking non-ideality effects. As discussed earlier, increasing the ambient pressure can directly influence the phase change and gas phase enthalpies, while QM enthalpies are computed for molecules in vacuo. We studied this direct impact of pressure on gas phase enthalpies via Eq. 5. However, this correction could only slightly improve the accuracy of 
Table 3 Comparison of QM evaluated enthalpies for different levels of theory.

\begin{tabular}{|c|c|c|c|c|}
\hline Compound & method & source & $\Delta \mathrm{H}_{\text {comb,QM,opt,gas }}(\mathrm{kJ} / \mathrm{mole})^{*}$ & $\Delta \mathrm{H}_{\mathrm{exp}}(\mathrm{kJ} / \mathrm{mole})^{* *}$ \\
\hline \multirow[t]{4}{*}{ Ethane } & PBEP86-D3/Def2QZVP & Present study & -1427.915 & -1426.895 \\
\hline & MP2 & 11 & -1422.7 & \\
\hline & B3LYP/6-311G(d,p) & 12 & -1284.36 & \\
\hline & TPSS-TPSS/6-311G+ +(df,pd) & 12 & -1275.44 & \\
\hline \multirow[t]{4}{*}{ Propane } & PBEP86-D3/Def2QZVP & Present study & -2041.343 & -2043.304 \\
\hline & MP2 & 11 & -2036.6 & \\
\hline & B3LYP/6-311G(d,p) & 12 & -1853.63 & \\
\hline & TPSS-TPSS/6-311G+ +(df,pd) & 12 & -1839.42 & \\
\hline \multirow[t]{4}{*}{ n-Butane } & PBEP86-D3/Def2QZVP & Present study & -2654.639 & -2655.470 \\
\hline & MP2 & 11 & -2650.2 & \\
\hline & B3LYP/6-311G(d,p) & 12 & -2423.40 & \\
\hline & TPSS-TPSS/6-311G+ +(df,pd) & 12 & -2403.74 & \\
\hline \multirow[t]{2}{*}{ Cyclopropane } & PBEP86-D3/Def2QZVP & Present study & -1959.477 & -1958.092 \\
\hline & MP2 & 11 & -1957.2 & \\
\hline \multirow[t]{2}{*}{ Cyclobutane } & PBEP86-D3/Def2QZVP & Present study & -2566.201 & -2566.498 \\
\hline & MP2 & 11 & -2566.0 & \\
\hline \multirow[t]{2}{*}{ Cyclohexane } & PBEP86-D3/Def2QZVP & Present study & -3685.178 & -3686.904 \\
\hline & MP2 & 11 & -3685.0 & \\
\hline \multirow[t]{7}{*}{ n-hexane } & PBEP86-D3/Def2QZVP & Present study & -3881.517 & -3883.785 \\
\hline & $\mathrm{HF} / 6-311++\mathrm{G}(3 \mathrm{df}, 3 \mathrm{pd})$ & 12 & -3389.77 & \\
\hline & B3LYP/6-311G(d,p) & 12 & -3563.42 & \\
\hline & B3LYP/6-311+ +G(3df,3pd) & 12 & -3765.90 & \\
\hline & B3LYP/6-311+ +G(3df,3pd) & 12 & -3737.44 & \\
\hline & PBEPBE/6-311+ +G(3df,3pd) & 12 & -3654.47 & \\
\hline & TPSS-TPSS/6-311G+ +(df,pd) & 12 & -3532.84 & \\
\hline \multirow[t]{6}{*}{ 1-pentanol } & PBEP86-D3/Def2QZVP & Present study & -3124.418 & -3122.477 \\
\hline & $\mathrm{HF}$ & 13 & -2688.45 & \\
\hline & MP2 & 13 & -3189.30 & \\
\hline & B3LYP & 13 & -2963.65 & \\
\hline & M06 & 13 & -3286.41 & \\
\hline & $\omega B 97 X-D$ & 13 & -2939.76 & \\
\hline
\end{tabular}


predicted combustion enthalpies, as can be seen in table 1. The more significant impact of the ambient pressure on gas phase enthalpies can be attributed to the formation of molecular clusters in the gas phase at high pressures. For example, for accurate evaluation of the phase change enthalpy as well as the saturation vapor pressure of water, it has been shown that clustering of two or further molecules in the gas phase should be taken into account [32]. Such gas phase clustering reduces the gas phase enthalpy compared to the in vacuo state, which is in line with the observed difference between the QM evaluated and optimized enthalpy of water, reported in table 2 .

In addition to the inaccuracies resulting from theoretical computations, systematic or operational errors in experimental data can also contribute to inconsistency between the theoretically evaluated and reference data. For example, we observed an on average 1.59 $\mathrm{kJ} / \mathrm{mol}$ absolute deviation in phase change enthalpies of our studied reactants between the NIST and DIPPR databases which results in the same deviation between the theoretically predicted gross combustion enthalpies calculated using each one of these two databases. Similar to the vaporization enthalpy, the experimentally determined combustion enthalpies also show some variations from different sources. For example, slight inaccuracy in measuring the combustion enthalpy of benzoic acid, which is used to calibrate the calorimeter [14], can result in a linearly distributed deviation among measured combustion enthalpies of all other compounds. That can be a potential reason of suitability of a linear curve fitting to empirically correcting the predicted combustion enthalpies.

\section{Summary and conclusion}

In summary, in the present study, we have discussed computational details which can result in high-accuracy evaluation of combustion enthalpy. To that end, the main considerations in theoretical computations should be directed towards selecting an appropriate level of theory and searching for structures with energies close to the global minimum energy, e.g. by multistart optimization. In reproducing the net heat of combustion, the phase change enthalpy of the reactants should be subtracted from the QM-evaluated gas phase enthalpies. For the gross heat of combustion, the vaporization enthalpy of water should also be subtracted from the QM-evaluated gas phase enthalpy of water. Accordingly, the inaccuracies in the mentioned phase change enthalpies as well as the experimental measurement of combustion enthalpy can also contribute to inconsistencies between the theoretically predicted and experimentally determined combustion enthalpies, as demonstrated in the present study. 


\section{Acknowledgment}

The author wish to thank Bernd Hartke in CAU university of Kiel for his fruitful discussions and reviewing this work.

\section{References:}

1. King, M. K.; Ross, H. D., Overview of the NASA microgravity combustion program. AIAA journal 1998, 36 (8), 1337-1345.

2. Suleyman A. Gokoglu, D. L. D., Dennis P. Stocker, Paul V. Ferkul, Sandy L. Olson, Michael C. Hicks A Researcher's Guide to: Combustion Science. NASA 2016.

3. Motil, B.; Urban, D., Combustion, Complex Fluids, and Fluid Physics Experiments on the ISS. 2012.

4. Zhu, Y.; Zhou, C.-W., Chemical kinetics study of 1, 3-butadiene+ HO2; implications for combustion modeling and simulation. Combustion and Flame 2020, 221, 241-255.

5. Kopp, W. A.; Kröger, L. C.; Döntgen, M.; Jacobs, S.; Burke, U.; Curran, H. J.; Heufer, K. A.; Leonhard, K., Detailed kinetic modeling of dimethoxymethane. Part I: Ab initio thermochemistry and kinetics predictions for key reactions. Combustion and Flame 2018, 189, 433-442.

6. Ye, L.; Zhang, L.; Qi, F., Ab initio kinetics on low temperature oxidation of iso-pentane: the first oxygen addition. Combustion and Flame 2018, 190, 119-132.

7. Shi, X.; Wang, Q.; Violi, A., Chemical pathways for the formation of benzofuran and dibenzofuran in combustion. Combustion and Flame 2020, 212, 216-233.

8. Shyamala, B.; Lal, S.; Chowdhury, A.; Namboothiri, I. N.; Kumbhakarna, N., Cubane decomposition pathways-A comprehensive study. Combustion and Flame 2018, 197, 111-119.

9. Osmont, A.; Catoire, L.; Gökalp, I.; Yang, V., Ab initio quantum chemical predictions of enthalpies of formation, heat capacities, and entropies of gas-phase energetic compounds. Combustion and Flame 2007, 151 (1-2), 262-273.

10. Swihart, M. T.; Catoire, L., Thermochemistry of aluminum species for combustion modeling from ab initio molecular orbital calculations. Combustion and flame 2000, 121 (1-2), 210-222.

11. Whyman, G.; Savoskin, M.; Yaroshenko, A.; Kapkan, L.; Popov, A., Straightforward ab initio calculation of enthalpies of combustion and formation of hydrocarbons. Journal of Molecular Structure: THEOCHEM 2003, 637 (1-3), 183-187.

12. Audran, G.; Marque, S. R.; Siri, D.; Santelli, M., Enthalpy of Combustion on n-Alkanes. Quantum Chemical Calculations up to n-C60H122 and Power Law Distributions. ChemistrySelect 2018, 3 (31), 9113-9120.

13. Mazzuca, J. W.; Downing, A. R.; Potter, C., Empirically corrected electronic structure calculations applied to the enthalpy of combustion physical chemistry laboratory. Journal of Chemical Education 2019, 96 (6), 1165-1170.

14. D4809-13, A., Standard Test Method for Heat of Combustion of Liquid Hydrocarbon Fuels by 
Bomb Calorimeter (Precision Method). ASTM International 2013.

15. Walters, R. N., Molar group contributions to the heat of combustion. Fire and materials 2002, 26

(3), 131-145.

16. McQuarrie, D. A., Statistical thermodynamics. 1973.

17. O’Boyle, N. M.; Banck, M.; James, C. A.; Morley, C.; Vandermeersch, T.; Hutchison, G. R., Open Babel: An open chemical toolbox. Journal of cheminformatics 2011, 3 (1), 1-14.

18. Kozuch, S.; Martin, J. M., DSD-PBEP86: in search of the best double-hybrid DFT with spincomponent scaled MP2 and dispersion corrections. Physical Chemistry Chemical Physics 2011, 13 (45), 20104-20107.

19. Alibakhshi, A.; Hartke, B., Improved prediction of solvation free energies by machine-learning polarizable continuum solvation model. Nature Communications 2021, 12 (1), 1-7.

20. Alibakhshi, A., Thermodynamically effective molecular surfaces for more efficient study of condensed-phase thermodynamics. 2021.

21. Alibakshi, A., Strategies to develop robust neural network models: prediction of flash point as a case study. Analytica chimica acta 2018, 1026, 69-76.

22. Hui, A. X. H., ENTHALPIES OF VAPORIZATION OF SOME MUITICHLORO-ALKANES. Acta Physico-chimica Sinica 1989, 05.

23. Murdock, J. W., Fundamental fluid mechanics for the practicing engineer. CRC Press: 2018.

24. Frisch, M.; Trucks, G.; Schlegel, H.; Scuseria, G.; Robb, M.; Cheeseman, J.; Scalmani, G.; Barone, V.; Petersson, G.; Nakatsuji, H., Gaussian 16. Revision A 2016, 3.

25. Goerigk, L.; Hansen, A.; Bauer, C.; Ehrlich, S.; Najibi, A.; Grimme, S., A look at the density functional theory zoo with the advanced GMTKN55 database for general main group thermochemistry, kinetics and noncovalent interactions. Physical Chemistry Chemical Physics 2017, 19 (48), 3218432215.

26. Kesharwani, M. K.; Brauer, B.; Martin, J. M., Frequency and zero-point vibrational energy scale factors for double-hybrid density functionals (and other selected methods): can anharmonic force fields be avoided? The Journal of Physical Chemistry A 2014, 119 (9), 1701-1714.

27. Riplinger, C.; Sandhoefer, B.; Hansen, A.; Neese, F., Natural triple excitations in local coupled cluster calculations with pair natural orbitals. The Journal of chemical physics 2013, 139 (13), 134101. 28. Dieterich, J. M.; Hartke, B., Error-safe, portable, and efficient evolutionary algorithms implementation with high scalability. Journal of chemical theory and computation 2016, 12 (10), 5226-5233.

29. Hartke, B., Wiley Interdiscip. Rev.: Comput. Mol. Sci 2011, 1, 879-887.

30. Dieterich, J. M.; Hartke, B., OGOLEM: Global cluster structure optimisation for arbitrary mixtures of flexible molecules. A multiscaling, object-oriented approach. Molecular Physics 2010, 108 (3-4), 279-291.

31. Pracht, P.; Bohle, F.; Grimme, S., Automated exploration of the low-energy chemical space with fast quantum chemical methods. Physical Chemistry Chemical Physics 2020, 22 (14), 7169-7192.

32. Parsafar, G. A.; Moeini, V.; NAJAFI, B., Pressure dependence of liquid vapor pressure: an improved gibbs prediction. 2001. 